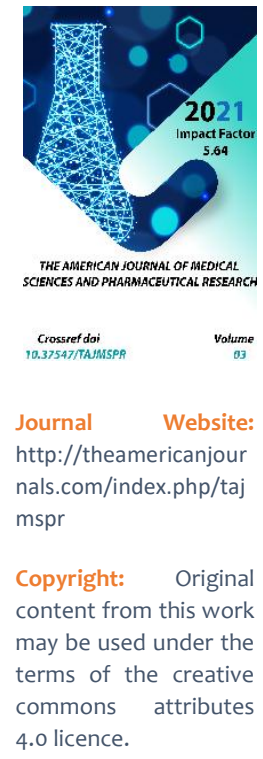

\title{
Influence Of Different Hypoglycemic Therapy Schemes On Carbohydrate Exchange Indicators In Type 2 Diabetes Mellitus
}

\author{
S.I. Ismailov \\ Department Of Endocrinology, Tashkent Pediatric Medical Institute, Tashkent, Uzbekistan \\ S.U. Muminova \\ Department Of Endocrinology, Tashkent Pediatric Medical Institute, Tashkent, Uzbekistan
}

\section{ABSTRACT}

Aim of the study: To study the effect of prescribing inhibitors of sodium glucose cotransporter type 2 (iSGLT-2) and inhibitors of dipeptidyl peptidase-4 (iDDP-4) on the parameters of carbohydrate metabolism in patients with type 2 diabetes.

Materials and methods: A prospective study included 80 patients with type 2 diabetes. The average age was $52.7 \pm 3.78$ years; diabetes experience -8 years; $\mathrm{BMI}-30 \pm 0.17$; Hb1C-9.2 $\pm 0.4 \%$; fasting glycemia $-10.2 \mathrm{mmol} / ;$ eGFR-78 ml/min; TG-2.7 \pm 0.44 ; total cholesterol-3.4 $\pm 0.72 ; \mathrm{MAU} 32 \pm 0.125$. The patients were divided into 2 groups: group 1 - 30 patients with DN with impaired renal function and 30 patients with diabetic nephropathy without renal dysfunction in the presence of metformin + iSGLT- 2; 2 group of 30 patients with impaired renal function and 30 patients with diabetic nephropathy without impaired renal function on the background of metformin + iDPP- 4 .

Results: The study of the effect of the inclusion of drugs iSGLT-2 (group 1) and iDPP-4 (group 2) showed a positive dynamics of carbohydrate metabolism indicators in patients with type 2 diabetes. So if the initial indicators in the groups were comparable in terms of glycemic control indicators, then by the $3 \mathrm{rd}$ month of treatment there was a significant decrease in HbA1C in the 1st group of patients in relation to the 2nd group. The result of the correction performed within 3 months was the achievement of the state of compensation in the 1 st group in $36.7 \%$, and in the 2nd group in $28.3 \%$, $48.3 \%$ of the patients of the 1 st group were brought into the state of sub compensation and $31.7 \%$ of patients of the 2 nd group.

Conclusion: On the combination of metformin and INGLT-2, a larger number of patients managed to achieve the set goals of therapy with a lower risk of overt hypoglycemia, then this combination should be considered not only more effective. 


\section{KEYWORDS}

Diabetes mellitus, diabetic nephropaty, hypoglycemic therapy.

\section{INTRODUCTION}

Diabetes mellitus (DM) is a global medical and social problem of our time, which is faced by medical science and healthcare in almost all countries of the world. World health organization (WHO) for the first time recognized this disease as a new noninfectious epidemic of the 21st century in relation to a chronic degenerative disease, since in recent years the growth rate of patients has become threatening. The urgency of diabetes mellitus is determined by its progressive morbidity $[1,2]$. Correction and prevention of the main manifestations of the disease and its complications are priority areas of medicine. The study of the patterns of the epidemiological status The development of the diabetes process, the solution of problems related to the increase in the prevalence of diabetes are numerous studies that are currently becoming a priority. The situation in Uzbekistan follows the global trend. According to 2019 data, 230610 patients with diabetes are registered in the country: 18349 patients with type 1 diabetes and 212261 - with type 2 diabetes. According to screening studies, the prevalence of type 2 diabetes in Uzbekistan over the past 14 years has increased 1.6 times and, according to the latest data (2015), is hypoglycemic therapy $7.9 \%$ among people over 35 years old $[1,3]$. Despite the variety of drugs, satisfactory glycemic control is achieved in no more than $40 \%$ of patients. The standard treatment regimen for type 2 diabetes, both in domestic and international recommendations, involves monotherapy with metformin at the onset of the disease (sometimes in combination with another drug for high hyperglycemia). The long-term management of type 2 diabetes was mainly aimed at glycemic control. A metaanalysis based on the results of 4 fundamental studies of diabetology (ACCORD, ADVANCE, UKPDS and VADT), which includes more than 27 thousand patients with type 2 diabetes, confirmed the primacy of intense glycemia for the prevention of chronic microvascular diseases of type 2 diabetes, including diabetic damage [4]. The pathogenetic multifactoriality and morphological heterogeneity of DN make one think about how universal the mechanisms of nephroprotection are. Taking into account the key role of hyperglycemia in the pathogenesis of $\mathrm{DN}$, in recent years, the nephroprotective properties of hypoglycemic agents have been actively studied, since, apparently, any drug that corrects hyperglycemia can have a moderate beneficial effect on the development of DN. However, it is still unclear whether different hypoglycemic drugs are equally effective in slowing the progression of renal dysfunction in diabetes mellitus [5]. Treatment of patients with $\mathrm{DN}$ in type 2 diabetes with the appointment of iDPP-4 promotes an increase in the level of endogenous GLP-1, improvement of its functioning, which in turn leads to the restoration of the function of GLP-1 receptors in the structures of the kidneys and vascular endothelium [6], reducing the effect of oxidative stress and suppresses excessive proliferation [7,8]. In addition, the multiple nephroprotective effects of iDPP-4 may possibly have a relationship with metamorphoses of the profile of the substrates of iDPP-4 action [9]. The circulating 
form of DPP-4 is involved in the degradation of neuroregulatory peptides, hormones, intermediates, and growth factors. DPP-4 has natriuretic properties, has an antiinflammatory effect, and also promotes vasodilation and direct protection of renal cells [10]. Over the past 2 decades, the pharmacotherapy of type 2 diabetes has been supplemented by several new classes of antihyperglycemic drugs (ADP) with different mechanisms of action. These classes of drugs, in addition to a positive effect on carbohydrate metabolism, have the ability to correct other metabolic disorders that are risk factors for the development and progression of type 2 diabetes [11,14]. Among them, inhibitors of sodium-glucose cotransporters type 2 (iSGLT-2), or gliflozins, are effective not only for their pronounced positive metabolic effects, but also for their ability to correct hemodynamic disorders, reducing intraglomerular hypertension and systemic arterial pressure, which can potentially have a nephroprotective effect. [12]. Thus, due to their unique mechanism of action, inhibitors of the sodium-glucose co-transporter type 2, in addition to glycemic and metabolic effects, have the ability to exert an inhibitory effect on various aspects of the pathogenesis of microand macro vascular complications of diabetes mellitus. Further large-scale clinical trials should provide information on which groups of patients with type 2 diabetes mellitus can benefit most from the prescription of gliflozin while minimizing the risk of adverse events[13,15].

\section{OBJECTIVE}

To study of the effect of prescribing inhibitors of sodium glucose cotransporter type 2 (iSGLT-2) and inhibitors of dipeptidyl peptidase-4 (iDDP-4) on the parameters of carbohydrate metabolism in patients with type 2 diabetes.

\section{MATERIAL AND METHODS}

A prospective study included 80 patients with type 2 diabetes the average age was $52.7 \pm$ 3.78 years, diabetes experience -8 years; BMI$30 \pm 0.17$; Hb1C-9.2 $\pm 0.4 \%$; fasting glycemia $10.2 \mathrm{mmol} / ;$ eGFR-78 ml/min; TG-2.7 \pm 0.44 ; total cholesterol-3.4 \pm 0.72 ; MAU $32 \pm 0.125$. To study the effect of various hypoglycemic therapy regimens on the functional state of the kidneys in type 2 diabetes mellitus, the patients were divided into 2 groups: group 1 30 patients with DN with impaired renal function and 30 patients with diabetic nephropathy without renal dysfunction in the presence of metformin + inhibitors. sodiumglucose cotransporter type 2 (iSGLT-2); 2 group of 30 patients with impaired renal function and 30 patients with diabetic nephropathy without impaired renal function on the background of metformin + dipeptidyl peptidase-4 inhibitors (iDPP-4). The diagnosis of diabetes and complications was confirmed by clinical and laboratory research methods and consultations of narrow specialists, according to the WHO classification. Clinical examination was carried out at the Republican Specialized Scientific-and-Practical Medical Centre of Endocrinology named after Academician Ya.Kh.Turakulov under the Ministry of Health of the Republic of Uzbekistan.. Clinical condition of patients physical examination (BMI, BP, PS).Examination of patients included: collection of anamnesis, complaints, consideration of concomitant pathology, constancy of drug treatment. An assessment of the compensation of type 2 diabetes was carried out, taking into account the selfcontrol of glycemia. The indicators of fasting glucose (after 8 hours of eating) and after 2 hours of starting to eat were taken into account.

Glycemia was determined on an automatic biochemical analyzer BS-380 "Mindray" by 
glucose oxidase method in venous blood on an empty stomach using HUMAN Glucose reagents (Germany). TSH) using kits from Cypress Diagnostics (Belgium). According to the WHO criteria (2003): Glycated hemoglobin (HbA1c) was determined by the using the HbA1c analyzer (automatic machine) Huma Nex A1c with HUMAN reagents (Germany).

\section{RESEARCH RESULTS AND DISCUSSION}

The study of the effect of the inclusion of drugs iSGLT-2 (group 1) and iDPP-4 (group 2) showed a positive dynamics of carbohydrate metabolism indicators in patients with type 2 diabetes. So if the initial indicators in the groups were comparable in terms of glycemic control indicators, then by the 3 rd month of treatment there was a significant decrease in $\mathrm{HbA1c}$ in the 1st group of patients in relation to the 2nd group. The decrease in $\mathrm{HbA} 1 \mathrm{c}$ in the 1st group occurred by $9.8 \%$ ( $9.2 \pm 0.4 \%$ versus $8.3 \pm 0.12 \% ; P<0.05)$, while in the 2 nd group by $7.9 \%(8.8 \pm 0.7 \%$ versus $8.1 \pm 0.6 \%$; $P>0.05)$ which is almost $2 \%$ lower.

The intergroup difference in the dynamics of treatment after 3 months was significant, as the percentage between the initial data and after 3 months in the 1st group was almost 2 times higher than the data of the 2nd group (24.9\% and $19.8 \%$ versus $14,1 \%$ vs. $16.8 \%$; P $<0.05)$. A similar trend was observed in the next 3 months. In the next 3 months, there is a decrease in fasting glycemia in the 1st group by $29.9 \%$, while in the 2nd group by $19.8 \%$, which is significantly low $(P<0.05)$. The level of postprandial glycemia within 6 months in both the 1st and 2nd groups significantly decreases in relation to the initial data, however, in the 1st group it is significantly pronounced - by $25.6 \%$ and by $19.9 \%(P<0.05)$. The content of HbA1C after 6 months decreased by $25 \%$ in the 1 st group of patients with diabetes mellitus 2 and by $10.2 \%$ in the 2nd group $(P<0.05)$. The reason for these differences is nocturnal hypoglycemia. The lack of flexibility in titrating the dose and taking each of the components of the drug separately leads to the fact that, in parallel with an increase in the dosage of metformin, the dosage of iDPP-4 also increases, as a result of which the hypoglycemic effect of the combination increases significantly and the frequency of hypoglycemia increases. It is because of the lower frequency of hypoglycemia on therapy with metformin and iSGLT-2 that a larger number of patients managed to achieve the goals of therapy both in terms of the level of glycated hemoglobin, fasting glycemia and 2 hours after a meal, and in terms of three indicators simultaneously both after 3 months and after 6 months. therapy. 
The proportion of patients (\%) in the groups that achieved the goals of therapy for the level of HbAlc, fasting glycemia, 2 hours after a meal and three indicators of glycemic control simultaneously.

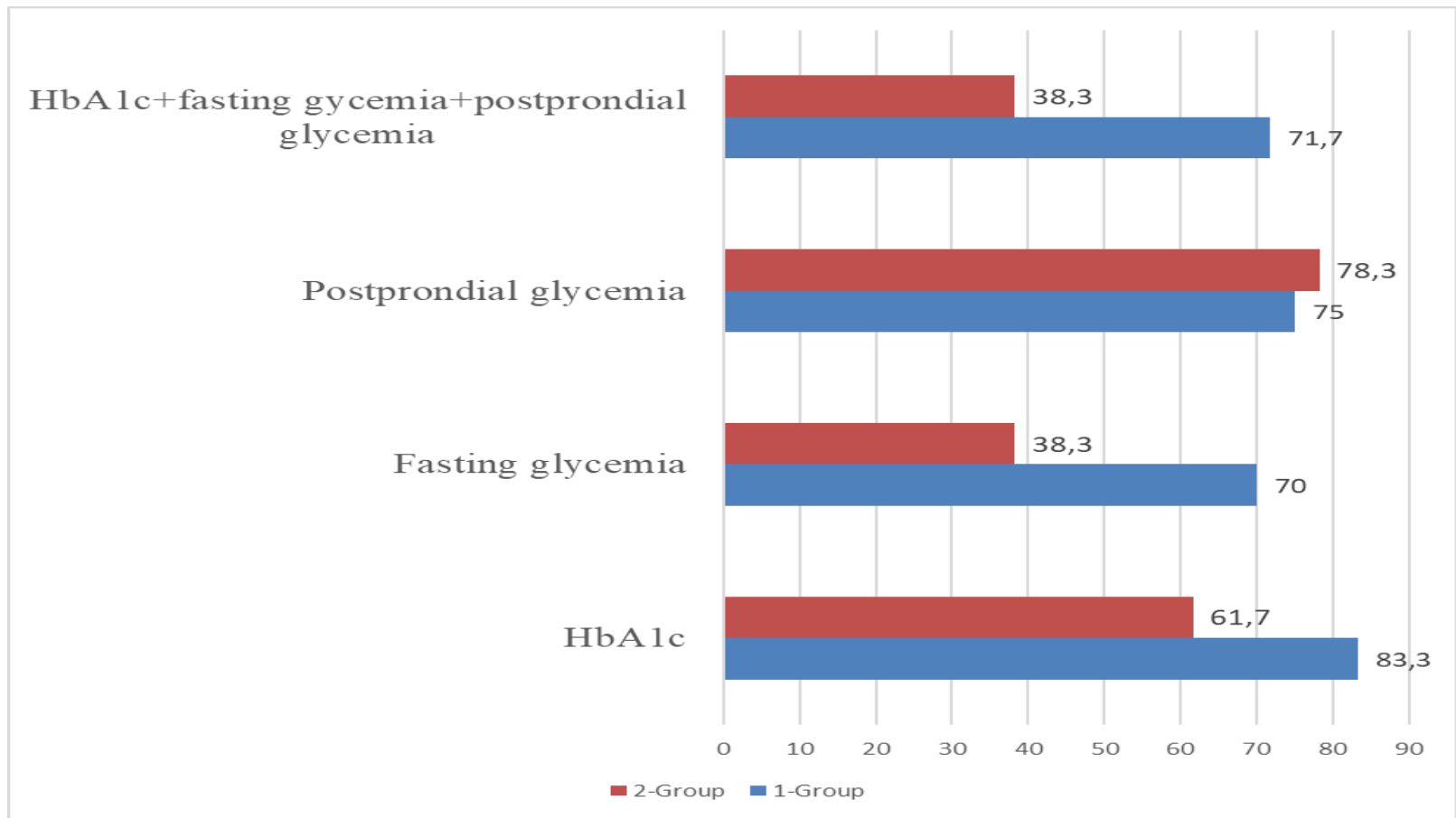

The result of the correction performed within 3 months was the achievement of the state of compensation in the 1st group in $36.7 \%$, and in the 2 nd group in $28.3 \%, 48.3 \%$ of the patients of the 1st group were brought into the state of subcompensation and $31.7 \%$ of patients of the 2nd group. The percentage of the occurrence of decompensation was higher in the 2nd group (40\%) in relation to the 1 st group (30\%). Over the next 3 months, the main indicators of carbohydrate metabolism continued to improve, which led to an increase in the number of patients who reached a state of compensation by the end of 6 months of the study in group 1 - in $60.0 \%$ of patients, and in the second group it was almost 2 times less - in $31.7 \%$ of patients ( $P$ $<0.05)$.

\section{CONCLUSION}

On the combination of metformin and iSGLT-2, a larger number of patients managed to achieve the set goals of therapy with a lower risk of overt hypoglycemia, then this combination should be considered not only more effective. Compensation of carbohydrate metabolism plays a key role in preventing the development and slowing the progression of CKD in patients with type 2 diabetes. In the late stages of CKD, the compensation of carbohydrate metabolism is extremely difficult due to the high risk of hypoglycemia due to a decrease in renal 
gluconeogenesis, accumulation of insulin, hypoglycemic agents and their metabolites, and the unreliability of $\mathrm{HbA1C}$ values in developing anemia. Thus, great care and an individual approach is required when choosing and intensifying antihyperglycemic therapy in patients with DN.

\section{REFERENCES}

1.

Alimov A.V., Khaidarova F.A., Berdykulova D.M., Alimova N.U., Sadikova A.S., Yuldasheva F.Z. Diabetes mellitus in the republic of uzbekistan: prevalence, morbidity according to statistical reports for the last 10 years. Bulletin of the Tashkent Medical Academy. - 2019. - No. 3. - P.. 8-13;

2. Mukhtarova Sh.Sh., Trigulova R.Kh., Ismailov S.I., Urmanova Yu.M., Influence of risk factors on the overall cardiovascular risk in patients with type 2 diabetes mellitus, Cadiological Journal of Uzbekistan, - 2020. - No. 1, P. - 69-74;

3. International Diabetes Federation. IDF diabetes atlas. 9th ed. Brussels: International Diabetes Federation; 2019.

4. Doo Soo Jeon. Paradigm Shift for the Treatment of Type 2 Diabetes Mellitus in Patients with Cardiovascular Disease: Cardiologist's Perspective. Cardiovasc Prev Pharmacother. 2020 Jan;2(1):11-17.

5. Thomas MC, Brownlee $M$, Susztak K, Sharma K, Jandeleit, Dahm KA, Zoungas $S$, et al. Diabetic kidney disease. Nat Rev Dis Primers 2015;1:15018.

6. Bae JH, Kim S, Park EG, Kim SG, Hahn $\mathrm{S}$, Kim NH. Effects of dipeptidyl peptidase-4 inhibitors on renal outcomes in patients with type 2 diabetes: a systematic review and meta-analysis. Endocrinol Metab (Seoul) 2019; 34: 80-92.

7. Makrilakis, Konstantinos. The Role of DPP-4 Inhibitors in the Treatment Algorithm of Type 2 Diabetes Mellitus: When to Select, what to Expect. International journal of environmental research and public health. 2019; vol. 16 (15) 2720.

8. Ide $M$, Sonoda $N$, Inoue $T$, et al. The dipeptidyl peptidase-4 inhibitor, linagliptin, improves cognitive impairment in streptozotocin-induced diabetic mice by inhibiting oxidative stress and microglial activation. PLoS One. 2020;15(2).

9. Kawanami D, Takashi Y, Takahashi H, Motonaga $\mathrm{R}$, Tanabe $\mathrm{M}$. Renoprotective Effects of DPP-4 Inhibitors. Antioxidants (Basel). 2021; 5;10(2):246.

10. Avogaro A, de Kreutzenberg S, Fadini G. Dipeptidyl-peptidase 4 inhibition: linking metabolic control to cardiovascular protection. Curr Pharm Des. 2014;20(14):2387-2394.

11. Davies MJ, D'Alessio DA, Fradkin J, Kernan WN, Mathieu C, Mingrone G, et al. Management of hyperglycemia in type 2 diabetes, 2018. A consensus report by the American Diabetes Association (ADA) and the European Association for the Study of Diabetes (EASD). Diabetes Care 2018;41:2669701.

12. Molecular markers of tubulointerstitial fibrosis and tubular cell damage in patients with chronic kidney disease [Electronic resource] / S.Nakagawa [et al.] // PLoS One. - 2015. - Vol. 10, № 8. - e0136994.

13. Trujillo J.M., Nuffer W.A. Impact of Sodium-Glucose Cotransporter 2 Inhibitors on Nonglycemic Outcomes in Patients with Type 2 Diabetes // 
The American Journal of Medical Sciences and Pharmaceutical Research

(ISSN - 2689-1026)

Published: June 16, 2021 | Pages: 13-19

Doi: https://doi.org/10.37547/TAJMSPR/Volume03Issue06-02

Pharmacotherapy. 2017. Vol. 37. № 4. Pp. 481-491. 25.;

14. Zaccardi F., Webb D., Htike Z., et al. Efficacy and safety of sodium-glucose co-transporter-2 inhibitors in type 2 diabetes mellitus: systematic review and network meta-analysis // Diabetes Obes. Metab., 2016. Vol. 18(8). Pp. 783794.

15. Trujillo J.M., Nuffer W.A. Impact of Sodium-Glucose Cotransporter 2 Inhibitors on Nonglycemic Outcomes in Patients with Type 2 Diabetes // Pharmacotherapy. 2017. Vol. 37. № 4. Pp. 481-491. 25.; 